\title{
Optical force microscopy with silicone rubber waveguides
}

\author{
S. Herminghaus ${ }^{\text {a) }}$ \\ Max Planck Institute for Colloid and Interface Science, Rudower Chaussee 5, \\ 12489 Berlin-Adlershof, Germany \\ M. Riedel and P. Leiderer ${ }^{\text {b) }}$ \\ Universität Konstanz, Fakultät für Physik, D-78434 Konstanz, Germany \\ M. Bastmeyer ${ }^{\mathrm{c})}$ and C. Stürmer \\ Universität Konstanz, Fakultät für Biologie, D-78434 Konstanz, Germany
}

(Received 15 August 1996; accepted for publication 5 November 1996)

\begin{abstract}
A simple technique is described for optically imaging the lateral distribution of normal forces exerted onto a flat surface. It is based on the detuning of a silicone rubber planar waveguide by the forces to be investigated. The method is demonstrated by imaging the contact line force of a sessile water droplet on the surface, with a force resolution better than $\mu \mathrm{N}$. It is shown that the lateral resolution may be much better than the decay length of the waveguide modes used.
\end{abstract}

The imaging of minute forces at surfaces and interfaces is presently a topic of great interest. This is due not only to the mere utilization of surface forces for imaging topographic features at surfaces, but also to the growing interest in quantitative investigations of "active" forces, such as those exerted by living cells adhering to a solid interface. ${ }^{1-3}$ With a scanning force microscope (SFM), which is the most commonly used device in the field of force imaging, the force exerted on a microscopically small cantilever (by its deflection) is measured at a single point of the sample at a time. ${ }^{4}$ However, in many experiments, it would be desirable to obtain a complete on-line image of the force distribution on a sample. ${ }^{3,5}$ In the present letter, we describe how such imaging may be achieved by using an optical planar waveguide made from a soft, clear rubber material. Since our technique is optical, the lateral resolution is not comparable to what is usually achieved with a SFM. However, the lateral resolution required depends strongly on the system under investigation. For instance, when wetting forces or cell adhesion forces are to be imaged, one need not necessarily strive for atomic resolution, and the smallness of the region scanned with the SFM may even be disadvantageous.

Our method is based on surface mode resonance microscopy, which has been described in many studies before. ${ }^{6,7}$ The sample, which is designed to support weakly damped optical eigenmodes such as surface plasmons or waveguide modes, is mounted on the base of a prism which is illuminated from inside under total internal reflection conditions. The incoming light is carefully collimated to give a parallel beam incident onto the sample at an angle of incidence which can be well controlled. The evanescent field leaking out of the prism base must be coupled appropriately to the sample. Whenever, and wherever on the sample, the in-plane wave number of the incoming light (which is determined by the angle of incidence) matches the propagation constant of an optical surface mode of the sample, this mode is resonantly excited at expense of the reflected light. Conse-

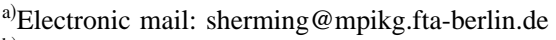

${ }^{b)}$ Electronic mail: paul.leiderer@uni-konstanz.de

${ }^{c)}$ Electronic mail: martin.bastmeyer@uni-konstanz.de
}

quently, regions of the sample, which provide a surface mode which is at resonance with the incoming light, will appear dark in reflection, while others will appear bright. Imaging the sample onto a CCD camera chip by means of a positive lens then yields a high contrast image of the spatial distribution of mode excitation on the sample.

The interface modes employed here are optical waveguide modes of a thin dielectric film (thickness $\approx$ a few microns), which are very sensitive to the optical properties of the waveguide material. Thus, if we choose a transparent rubber as the waveguide material, forces exerted on the sample (and, consequently, on the rubber layer) will detune the waveguide modes since they change the thickness and (by the Clausius-Mossotti relation) the refractive index of the waveguide. This results in a detectable image contrast when the sample is illuminated at an angle close to a waveguide mode resonance.

Our waveguides were prepared from poly(dimethylsiloxane) (M 12500 purchased from Sigma), which was spread on a metallized glass substrate for approximately 10 min using a spin coater at $7000 \mathrm{rpm}$. This yielded silicone oil films with a typical thickness of $6 \mu \mathrm{m}$. The substrates were glass slides coated with a 46-nm-thick evaporated silver film serving for optical coupling of the waveguide to the glass substrate. ${ }^{7-9}$ After spin casting, the films were mildly cross linked by irradiation with a 75 Watts Xenon UV lamp (LOT/ Osram, XBO 75) for $25 \mathrm{~min}$. The infrared components of the spectrum have been blocked by means of a 5 -cm-thick water cell, and the output beam had a diameter of $2 \mathrm{~cm}$ at the position of the sample. This procedure resulted in a waveguide material of high mechanical susceptibility with a certain remaining ability to flow, as could be judged from its imaging properties (see below).

In order to ensure optical separation of the waveguide from the adjacent sample space, it was necessary to evaporate a second silver layer atop of the silicone film. It turned out, however, that this was not easily possible for films that were merely UV-cross linked. The preparation of a stable, compact silver overlayer required the presence of a firmly cross linked topmost rubber layer, which could be generated by treating the waveguide with a glow discharge in a com- 


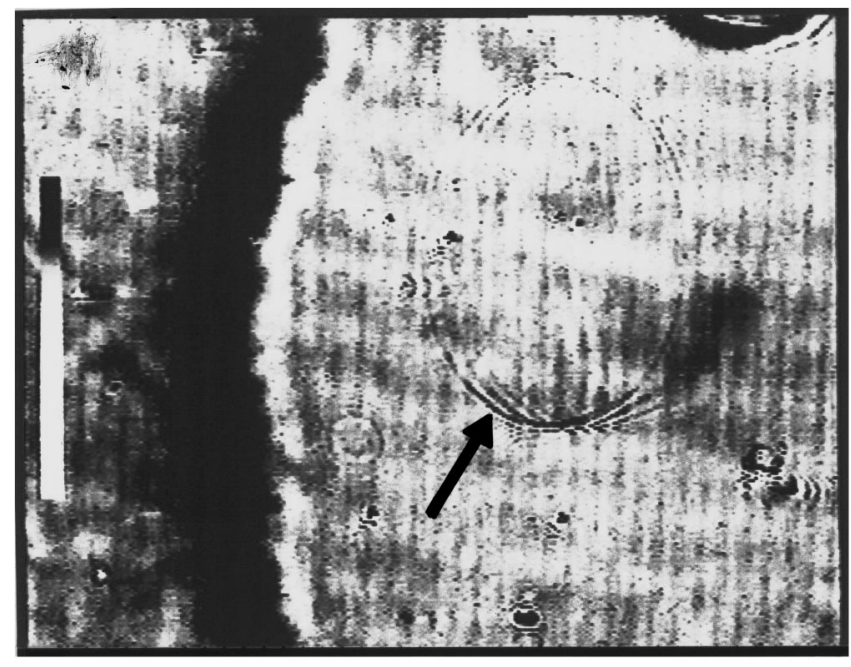

FIG. 1. Waveguide microscopy image of a silicone rubber waveguide (length of the grey level scale bar: $1.4 \mathrm{~mm}$ ). Regions of resonant excitation of waveguide modes appear as black features. They represent contour lines of the waveguide thickness, which is about $5.3 \mu \mathrm{m}$ in the shown region. A sessile water droplet is visible via its impact on the waveguide modes (arrow). The elliptical shape of the contact line is due to the tilted position of the sample with respect to the optical axis.

mercial sputter coating apparatus $(0.15$ Torr of air, $1.2 \mathrm{kV}$ discharge voltage for $5 \mathrm{~s}) .{ }^{10}$ After this treatment, a silver overlayer with a thickness of $100 \mathrm{~nm}$ was evaporated onto the silicone rubber. The so prepared sample was mounted with index matching fluid onto the base of a glass prism for performing waveguide mode microscopy.

Figure 1 shows a waveguide mode image of the sample obtained with $p$-polarized light at 45 deg internal angle of incidence. Light from a white light source, filtered by a 633 $\mathrm{nm}$ interference filter and a polarizer, has been used for illumination. The dark bar represents a region of resonant excitation of a waveguide mode. It represents a contour line of the waveguide thickness, the irregular shape stemming from local variations of the waveguide layer thickness. Also visible (arrow) is an elliptical feature with a diameter approximately equal to the length of the grey level scale bar. This represents the contact line of a sessile water droplet which had been placed onto the sample surface, and whose diameter was $1.5 \mathrm{~mm}$. While the actual contact line is circular, its image appears elliptical due to the tilted position of the sample with respect to the optic axis. ${ }^{7}$

At most parts of the contact line image, an interference pattern is visible, appearing as a multiple image of the contact line. This is a well established effect in surface resonance microscopy ${ }^{11}$ and stems from the interference of the incoming light with the waveguide modes in the nonresonant (bright) region, which are launched at discontinuities like the deformation of the waveguide below the contact line of the drop. The lateral extension of this pattern corresponds to the decay length of the waveguide mode, which is obviously much larger than the lateral resolution with which features like the contact line can be resolved. A complete theoretical treatment of the question of how small features can be resolved requires the numerical solution of the inhomogeneous wave equation with the structure to be resolved being represented by the (spatially varying) coefficient of the optical
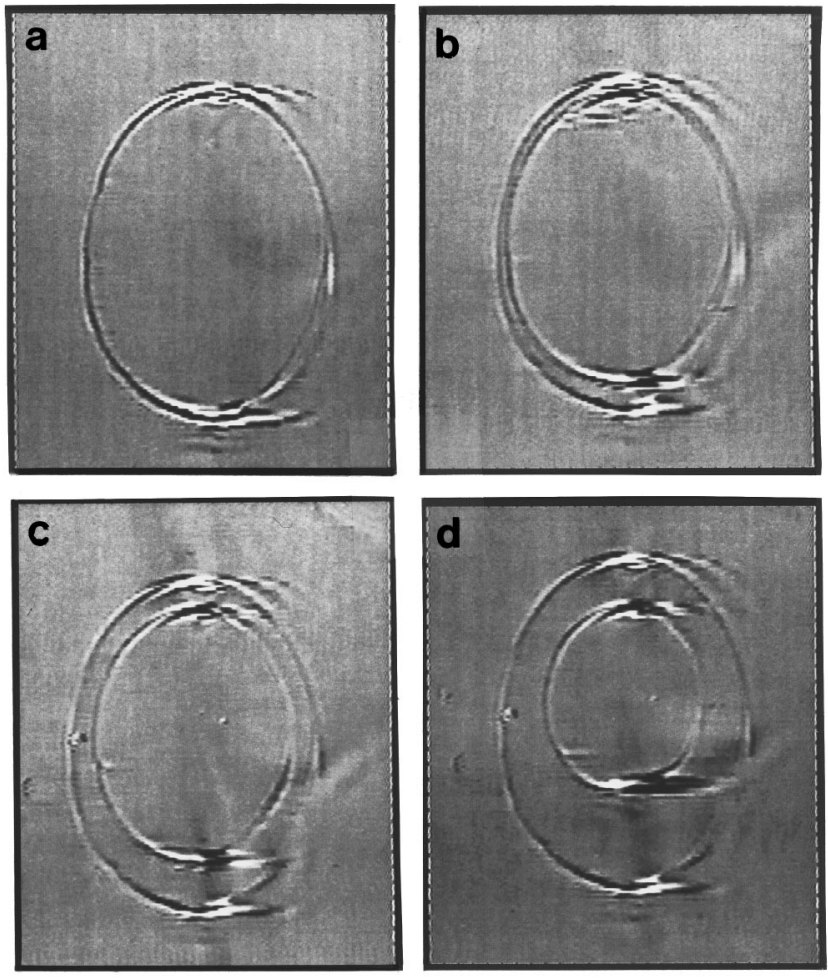

FIG. 2. Series of images obtained by image subtraction while the water droplet slowly evaporates. The drop exerts a force along its contact line, which amounts to $72 \mathrm{mN} / \mathrm{m}$ corresponding to the surface tension of water. The direction of illumination (and, consequently, of propagation of the waveguide modes) is from left to right.

field. This should describe the observed grey level profiles in detail and will be presented in a forthcoming publication.

The fact that there is no noticeable difference between the regions which are covered by water and those which are not, shows that sufficient optical separation between the waveguide and the adjacent half space is achieved by the top silver layer. It is now instructive to watch what happens when the water droplet slowly evaporates. This is shown in Figs. 2(a)-2(d), which have been obtained by on-line subtraction of the obtained images. Shortly after Fig. 1 was taken, the angle of incidence was adjusted so as to yield a waveguide resonance at the center of the drop, and the image was saved. All later images (Fig. 2) have been obtained by subtraction from this image. As a result, one can see that at those regions of the drop circumference which fulfill the waveguide resonance condition (those with horizontal tangent, in our case), the contrast is substantially enhanced, as compared to the others which are out of resonance.

When the droplet shrinks due to evaporation, the inverse of the original contact line image is left behind at the starting position due to the subtraction procedure. At the same time, one can see the smaller contact line circle emerge and shrink. As it can be judged from the equality of the grey levels inside and outside the outer ring, the deformation of the waveguide is completely reversible. Since the contact angle of the droplet was close to $\pi / 2$, the force exerted onto the sample at the contact line is given by the surface tension of water, which is $\sigma=72 \mathrm{mN} / \mathrm{m}$. Consequently, the total force exerted along the droplet circumference is $340 \mu \mathrm{N}$.

The excess pressure at the substrate-water interface is 


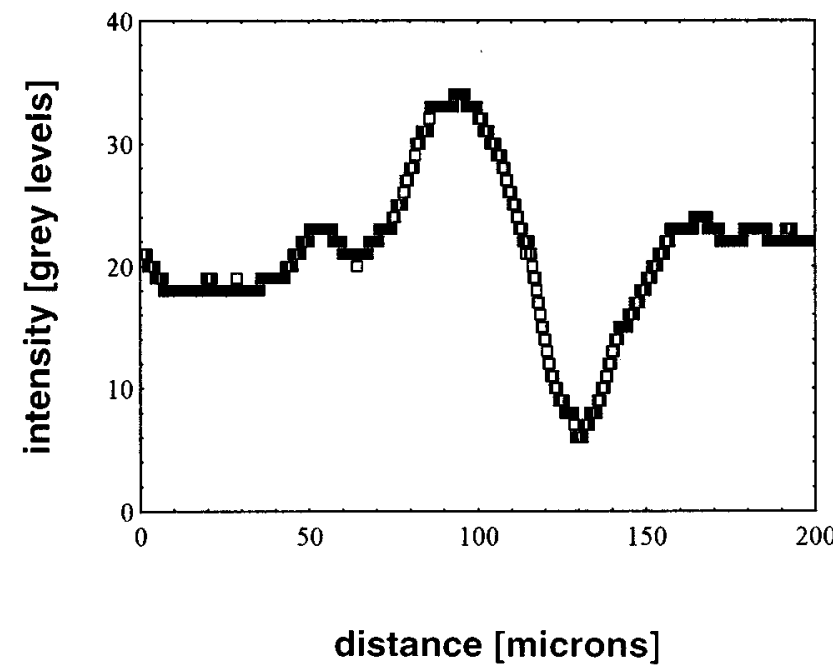

FIG. 3. Intensity profile across the contact line, as obtained from the grey levels of a subtracted image (cf. Fig. 2). The full scale in reflectance (100\%) corresponds to 255 grey levels.

$\Delta p=\sigma / r$ and diverges as the droplet radius $r$ approaches zero. Nevertheless, no noticeable change in grey value is observed within the droplet (i.e., the inner ring in Fig. 2) even at the very latest stage when its radius was only of the order of the width of the contact line image. This suggests that there is only a little sensitivity to homogeneously exerted forces, in contrast to lateral force gradients as they are present in the vicinity of the contact line. This supports our view that the film is only mildly cross linked, with a considerable remaining ability to flow. For further studies, it will thus be important to determine the spatio-temporal response function of the waveguide.

A typical cross section of the contact line image is presented in Fig. 3, which was taken through a nonresonant part of the contact line. The lateral extension of the perturbation is about $50 \mu \mathrm{m}$, in contrast to the actual extension of the contact line which is expected to be in the nm range (i.e., the typical range of wetting forces). ${ }^{12}$ This again suggests that there is considerable lateral transport of material (noncross linked PDMS) within the waveguide layer, which causes the observed broadening of the feature. If we take the inherent lateral resolution of the imaging process to be roughly 50 $\mu \mathrm{m}$ as suggested by Fig. 3, the signal displayed in the figure (30 grey levels peak to valley) corresponds to a local force of
3.6 $\mu \mathrm{N}$. Since the amplitude of the erratic (noiselike) parts of the profile is less than 3 grey levels, we can estimate that the local force resolution of the present set up is better than 360 $\mathrm{nN}$, which corresponds to a pressure of $1.4 \times 10^{-10} \mathrm{~N} / \mu \mathrm{m}^{2}$. This is within the range of typical cell adhesion forces. ${ }^{1}$

If one considers those regions of the contact line which are close to the waveguide resonance, one has a considerably higher force resolution. This is suggested by Fig. 2 which shows strongly enhanced features where one is close to the waveguide resonance (in Fig. 2, where the contact line has a horizontal tangent). However, as one can see from the effect of smearing in the direction of waveguide mode propagation, the lateral resolution is inferior at these places. One thus has an adjustable trade off between a high lateral resolution or a high force resolution, between which the system can be easily switched by changing the angle of incidence of the light.

In conclusion, we have introduced a novel technique of surface force imaging based on the excitation of waveguide modes in a soft rubber optical planar waveguide. Although considerable characterizational effort will be necessary in order to enable quantitative, accurate force measurements, the method seems well suited for a wide range of experiments on lateral force distributions in both static and, in particular, dynamic surface phenomena.

Fruitful discussions with G. Hertkorn, K. Phelan, and K. Jacobsen are gratefully acknowledged. This work was supported by the Deutsche Forschungsgemeinschaft.

${ }^{1}$ M. Opas, in: Cytomechanics, edited by Bereiter-Hahn, Anderson, and Reif (Springer, New York, 1987), pp. 274-285.

${ }^{2}$ K. L. Sung, P. Kuhlmann, and A. A. Brian, J. Cell. Sci. 103, 259 (1992).

${ }^{3}$ J. Lee, T. Oliver, M. Leonard, and K. Jacobsen, J. Cell. Biol. 127, 1957 (1994).

${ }^{4}$ D. E. Leckband, J. N. Israelachvili, F. J. Schmitt, and W. Knoll, Science 255, 1419 (1992); U. Dammer, P. Wagner, H. J. Güntherodt, and G. N. Misevic, Science 267, 1173 (1995).

${ }^{5}$ T. Oliver, J. Lee, and K. Jacobsen, Seminars in Cell Biology, 5, 139 (1994).

${ }^{6}$ W. Hickel, D. Kamp, and W. Knoll, Nature (London) 339, 186 (1989).

${ }^{7}$ For a review see, W. Knoll, Mater. Res. Soc. Symp. Proc. XVI, 29 (1991).

${ }^{8}$ J. R. Sambles, G. W. Bradbery, and F. Yang, Contemp. Phys. 32, 173 (1991).

${ }^{9}$ S. Herminghaus, M. Klopfleisch, and H. J. Schmidt, Opt. Lett. 19, 293 (1994).

${ }^{10}$ T. Oliver, M. Dembo, and K. Jacobsen, Cell Motil. Cytosk. 31, 225 (1995).

${ }^{11}$ B. Rothenhäusler and W. Knoll, J. Opt. Soc. Am. 5, 1401 (1988).

${ }^{12} \mathrm{M}$. Schick, in: Liquids at Interfaces, session XLVII, Les Houches, edited by J. Charvolin, J. F. Joanny, and J. Zinn-Justin (North-Holland, Amsterdam, 1988). 